\title{
Cellular- and histopathological effects of a pollutant gradient - summary
}

\author{
M. N. Moore* \\ Plymouth Marine Laboratory (West Hoe), Prospect Place, The Hoe, Plymouth PL1 3DH, United Kingdom
}

\section{INTRODUCTION}

The aim of the GEEP Workshop studies of cellular pathobiology was to assess the use of quantitative indices of subcellular and cellular perturbation in relation to the impact of environmental xenobiotics, both in the field and following experimental exposure A secondary objective was to try to relate any observed perturbations to functional alterations in cellular processes. The aim of the histopathology was to describe tissue and organ condition for evidence of xenobiotic impact and to relate this to animal health and to subcellular and cellular effects. All these studies were carried out on mussels Mytilus edulis and some on winkles Littorina littorea.

\section{REPRODUCTIVE CONDITION}

Quantitative histological analysis of the reproductive tissues in Mytilus edulis indicated gametogenic activity in both field and mesocosm samples. However, in mussels from field sites, the proportion of storage tissue was relatively high in relation to volume density of gametes. This indicated that reproductive activity was not dominant and was unlikely to interfere with the interpretation of environmental effects. In contrast some experimental mesocosm conditions revealed evidence of extensive reproductive activity, with the low exposure treatment having the least proportion of gametes. This factor may have contributed to these mussels having a higher lysosomal membrane stability. The high exposure treatment and its control were quite different from the other 3 treatments indicating that with regard to cellular condition, the high exposure treatment cannot realistically be compared with the

\footnotetext{
- This summary was written following discussions amongst all contributors to this section
}

low and medium exposures. The high exposure treatment was the only one that displayed evidence of xenobiotic-induced degeneration of gametes.

\section{CELLULAR PATHOBIOLOGY}

Lysosomal data indicated that Mytilus edulis from Field sites 2, 3 and 4 were impacted by pollutants There was good agreement between authors and between the various lysosomal characteristics tested Lysosomal enlargement, increased fragility, lipid accumulation and lipofuscin accumulation all emerged as good descriptors of pathological effects in the digestive cells. The mussel data were further supported by the evidence of increased lysosomal membrane fragility in the digestive cells of Littorina littorea from Sites 2, 3 and 4. These types of pathological alteration were interpreted as being indicative of augmented autophagocytosis resulting in increased catabolism of macromolecules and ultimately in cellular atrophy. This latter consequence was supported, in part, by evidence of tubule degeneration at Site 3.

The picture that emerged from the experimental mesocosm investigation was less clearcut, with mussels from all treatments showing evidence of cellular perturbation, probably induced by relative shortage of food within the mesocosm basins. However, when the high-exposure treatment was compared with its control there was good agreement between the authors that this condition was impacted by pollutants, in terms of lysosomal disturbance and cellular degeneration.

NADPH-fernhemoprotein reductase appeared to be a good indicator of xenobiotic effects, being directly correlated with the tissue concentrations of polycyclic aromatic hydrocarbons (PAHs). The specific cause of this induction of activity was not identified, but previous field and experimental investigations have indicated that a range of PAHs are capable of stimulating the activity of this enzyme. 


\section{HISTOPATHOLOGY}

Examination of Mytilus edulis from the field sites indicated that Sites 2, 3 and 4 were impacted. This was based on evidence of increased incidences of granulocytomas, aggregations of brown cells, digestive tubule dilation and degeneration. In mussels from the experimental treatments there was good agreement between authors that the high exposure condition was the most severely impacted. This was based on evidence of digestive tubule dilation and degeneration, as well as degenerative changes in the gills and kidney.

The incidence of parasitism in mussels from the field sites indicated that Site 1 was more affected than the other sites. Authors data coincided on this factor but did not give any indications regarding the cause of differences. It may be that mussels already impacted by xenobiotics are more prone to mortality if infected by parasites, or alternatively, other factors such as contact with intermediate or definitive hosts may be reduced at the impacted sites.

\section{CONCLUSIONS}

The results obtained by all 3 authors showed good general agreement in the assessment of effects in both the field (Table 1) and mesocosm samples. Furthermore, in instances where there was overlap in the variables being measured, the level of agreement was very close.

When the various hierarchical levels are considered - molecular, subcellular, cellular, tissue and organ some effects observed at the lower levels can be used to predict those observed at higher levels of organisation. For instance, on the basis of the increased fragility of the lysosomal membrane in the digestive cells of impacted animals, one would predict that lysosomal dysfunction would lead to increased autophagocytosis and increased vacuolar fusion as evidenced by
Table 1. Mytilus edulis. Ranking of field sites based on main cellular variables measured. (Site 1 is the reference site)

\begin{tabular}{|ll|}
\hline Variable & Site ranking \\
\hline NADPH-ferrihemoprotein reductase & $1=2<3=4$ \\
Lysosomal membrane stability & $1>4>2=3$ \\
Lysosomal enlargement & $1=4<2=3$ \\
Lysosomal and cytoplasmic lipid & $1<2=3=4$ \\
Lysosomal lipofuscin & $1<2=3=4$ \\
Brown staining lysosomes & $1>3=4$ \\
Pyramidal cell vacuolation & $1<2=3=4$ \\
Tubule degeneration & $1<3$ \\
Tubule dilation & $1=3<2=4$ \\
Granulocytomas & $1<3$ \\
Aggregations of brown cells & $1<3=4$ \\
Parasitism & $1>2=3=4$ \\
\hline
\end{tabular}

lysosomal enlargement, accumulation of lipid and lipofuscin. This would in turn lead to the observed atrophy of the digestive cells resulting in the dilation and degeneration of digestive tubules. The consequence of degeneration of the digestive tubules would be the failure of digestive and storage functions in the digestive gland.

The findings indicate that the relationships between many of the observed pathological effects and the level of contaminants are not necessarily simple. In a number of instances the most heavily contaminated mussels were not the most impacted, and this may be indicative of either interactive effects in complex mixtures of contaminants and/or the selection of more resistant forms at the most contaminated site.

The techniques used to investigate cellular and tissue effects are in general fairly straightforward, but the level of both structural and functional information obtained is high. That effects observed at one level of cellular organization can be used to predict those observed at higher levels is good confirmation that the mechanistic assumptions are firmly based. As such, this strengthens the case for the use of many of these types of methods in monitoring for the biological effects of environmental pollutants. 